\title{
Some remarks on a paper of V. A. Liskovets
}

\author{
László Tóth * \\ Department of Mathematics, University of Pécs \\ Ifjúság u. 6, H-7624 Pécs, Hungary \\ and \\ Institute of Mathematics, Department of Integrative Biology \\ Universität für Bodenkultur, Gregor Mendel-Straße 33, A-1180 Wien, Austria \\ E-mail: ltoth@gamma.ttk.pte.hu
}

\begin{abstract}
We deduce new properties of the orbicyclic function $E$ of several variables investigated in a recent paper by V. A. Liskovets. We point out that the function $E$ and its connection to the number of solutions of certain linear congruences occur in the literature in a slightly different form. We investigate another similar function considered by Deitmar, Koyama and Kurokawa by studying analytic properties of some zeta functions of Igusa type. Simple number theoretic proofs for some known properties are also given.
\end{abstract}

\section{Introduction}

In a recent paper Liskovets [10] investigated arithmetical properties of the function

$$
E\left(m_{1}, \ldots, m_{r}\right):=\frac{1}{M} \sum_{k=1}^{M} c_{m_{1}}(k) \cdots c_{m_{r}}(k),
$$

where $m_{1}, \ldots, m_{r}, M \in \mathbb{N}:=\{1,2, \ldots\}(r \in \mathbb{N}), m:=\operatorname{lcm}\left[m_{1}, \ldots, m_{r}\right], m \mid M$ and $c_{n}(k)$ is the Ramanujan sum defined as the sum of $k$-th powers of the primitive $n$-th roots of unity $(k, n \in \mathbb{N})$, i.e.,

$$
c_{n}(k):=\sum_{\substack{1 \leq j \leq n \\ \operatorname{gcd}(j, n)=1}} \exp (2 \pi i j k / n) .
$$

The function $E$ given by (1) has been introduced by Mednykh and Nedela [13] in order to handle certain problems of enumerative combinatorics. The function (1) has also arithmetical and topological applications and it is called in [10] as the "orbicyclic" arithmetic function.

For example, $E\left(m_{1}, \ldots, m_{r}\right)$ is the number of solutions $\left(x_{1}, \ldots, x_{r}\right) \in \mathbb{Z}_{M}^{r}$ of the congruence

$$
x_{1}+\ldots+x_{r} \equiv 0(\bmod M)
$$

satisfying $\operatorname{gcd}\left(x_{1}, M\right)=M / m_{1}, \ldots, \operatorname{gcd}\left(x_{r}, M\right)=M / m_{r}$, see [13, Lemma 4.1]. It follows from this interpretation that all the values $E\left(m_{1}, \ldots, m_{r}\right)$ are nonnegative integers.

Note that in case of one, respectively two variables,

$$
E\left(m_{1}\right):=\frac{1}{M} \sum_{k=1}^{M} c_{m_{1}}(k)= \begin{cases}1, & m_{1}=1 \\ 0, & \text { otherwise }\end{cases}
$$

${ }^{*}$ The author gratefully acknowledges support from the Austrian Science Fund (FWF) under the project Nr. P20847-N18. 


$$
E\left(m_{1}, m_{2}\right):=\frac{1}{M} \sum_{k=1}^{M} c_{m_{1}}(k) c_{m_{2}}(k)= \begin{cases}\phi(m), & m_{1}=m_{2}=m, \\ 0, & \text { otherwise }\end{cases}
$$

where $\phi$ is Euler's function. Formulae (3) and (4) are well known properties of the Ramanujan sums, (4) being their orthogonality property leading to the Ramanujan expansions of arithmetical functions, see for example [19].

Another function, similar to $E$, is

$$
A\left(m_{1}, \ldots, m_{r}\right):=\frac{1}{m} \sum_{k=1}^{m} \operatorname{gcd}\left(k, m_{1}\right) \cdots \operatorname{gcd}\left(k, m_{r}\right),
$$

where $m_{1}, \ldots, m_{r} \in \mathbb{N}(r \in \mathbb{N})$ and $m:=\operatorname{lcm}\left[m_{1}, \ldots, m_{r}\right]$, as above.

The function (5) was mentioned by Liskovets [10, section 4] and it was considered by Deitmar, Koyama and Kurokawa [9] in case $m_{j} \mid m_{j+1}(1 \leq j \leq r-1)$ by studying analytic properties of some zeta functions of Igusa type. The explicit formula for the values $A\left(m_{1}, \ldots, m_{r}\right)$ derived in [9, Section 3] was reproved by Minami [14] for the general case $m_{1}, \ldots, m_{r} \in \mathbb{N}$, using arguments of elementary probability theory. We remark that the corresponding formulae of both papers $[9,14]$ contain misprints.

For $r=1$ (5) reduces to the function

$$
A(m):=\frac{1}{m} \sum_{k=1}^{m} \operatorname{gcd}(k, m)=\sum_{d \mid m} \frac{\phi(d)}{d}
$$

giving the arithmetic mean of $\operatorname{gcd}(1, m), \ldots, \operatorname{gcd}(m, m)$. For arithmetic and analytic properties of $(6)$ and for a survey of other gcd-sum-type functions of one variable see Tóth [22].

In the present paper we deduce new properties of the functions $E$ and $A$ and use them to give simple number theoretic proofs for some of their known properties. We derive convolution-type identities for $E$ and $A$ (Propositions 3 and 12) to show that they are multiplicative as functions of several variables. We give other identities for $E$ and $A$ (Propositions 9 and 14) to obtain explicit formulae for their values. We consider common generalizations of these functions and point out that the function $E$ and its connection to the number of solutions of certain linear congruences occur in the literature in a slightly different form.

As an application of the identity of Proposition 3 we give a simple direct proof of the orthogonality property (4) of the Ramanujan sums (Application 8).

\section{Preliminaries}

We present in this section some basic notions and properties needed throughout the paper. For the prime power factorization of an integer $n \in \mathbb{N}$ we will use the notation $n=\prod_{p} p^{\nu_{p}(n)}$, where the product is over the primes $p$ and all but a finite number of the exponents $\nu_{p}(n)$ are zero.

We recall that an arithmetic function of $r$ variables is a function $f: \mathbb{N}^{r} \rightarrow \mathbb{C}$, notation $f \in \mathcal{F}_{r}$. If $f, g \in \mathcal{F}_{r}$, then their convolution is defined as

$$
(f * g)\left(m_{1}, \ldots, m_{r}\right)=\sum_{d_{1}\left|m_{1}, \ldots, d_{r}\right| m_{r}} f\left(d_{1}, \ldots, d_{r}\right) g\left(m_{1} / d_{1}, \ldots, m_{r} / d_{r}\right) .
$$

The set $\mathcal{F}_{r}$ forms a unital ring with ordinary addition and convolution (7), the unity being the function $\varepsilon^{(r)}$ given by

$$
\varepsilon^{(r)}\left(m_{1}, \ldots, m_{r}\right)= \begin{cases}1, & m_{1}=\ldots=m_{r}=1 \\ 0, & \text { otherwise. }\end{cases}
$$

A function $f \in \mathcal{F}_{r}$ is invertible iff $f(1, \ldots, 1) \neq 0$. The inverse of the constant 1 function is given by $\mu^{(r)}\left(m_{1}, \ldots, m_{r}\right)=\mu\left(m_{1}\right) \ldots \mu\left(m_{r}\right)$, where $\mu$ is the Möbius function.

A function $f \in \mathcal{F}_{r}$ is said to be multiplicative if it is nonzero and

$$
f\left(m_{1} n_{1}, \ldots, m_{r} n_{r}\right)=f\left(m_{1}, \ldots, m_{r}\right) f\left(n_{1}, \ldots, n_{r}\right)
$$


holds for any $m_{1}, \ldots, m_{r}, n_{1}, \ldots, n_{r} \in \mathbb{N}$ such that $\operatorname{gcd}\left(m_{1} \cdots m_{r}, n_{1} \cdots n_{r}\right)=1$.

If $f$ is multiplicative, then it is determined by the values $f\left(p^{a_{1}}, \ldots, p^{a_{r}}\right)$, where $p$ is a prime and $a_{1}, \ldots, a_{r} \in \mathbb{N}_{0}:=\{0,1,2, \ldots\}$. More exactly, $f(1, \ldots, 1)=1$ and for any $m_{1}, \ldots, m_{r} \in \mathbb{N}$,

$$
f\left(m_{1}, \ldots, m_{r}\right)=\prod_{p} f\left(p^{\nu_{p}\left(m_{1}\right)}, \ldots, p^{\nu_{p}\left(m_{r}\right)}\right) .
$$

For example, the functions $\left(m_{1}, \ldots, m_{r}\right) \mapsto \operatorname{gcd}\left(m_{1}, \ldots, m_{r}\right)$ and $\left(m_{1}, \ldots, m_{r}\right) \mapsto \operatorname{lcm}\left[m_{1}, \ldots, m_{r}\right]$ are multiplicative. The function $\mu^{(r)}$ is also multiplicative.

The convolution (7) preserves the multiplicativity of functions. Moreover, the multiplicative functions form a subgroup of the group of invertible functions with respect to convolution (7).

These properties, which are well known in the one variable case, follow easily from the definitions. For further properties of arithmetic functions of several variables and of their ring we refer to [2], [20, Ch. VII].

In the one variable case $\mathbf{1}, \operatorname{id}_{k}(k \in \mathbb{N}), \varepsilon$ and $\tau$ will denote the functions given by $\mathbf{1}(n)=1, \operatorname{id}_{k}(n)=n^{k}$ $(n \in \mathbb{N}), \varepsilon(1)=1, \varepsilon(n)=0$ for $n>1$ and $\tau=\mathbf{1} * \mathbf{1}$ (divisor function), respectively.

The Ramanujan sums $c_{n}(k)$ can be represented as

$$
c_{n}(k)=\sum_{d \mid \operatorname{gcd}(k, n)} d \mu(n / d) \quad(k, n \in \mathbb{N}) .
$$

Note that the function $n \mapsto c_{n}(k)$ is multiplicative for any fixed $k \in \mathbb{N}$, and for any prime power $p^{a}$ $(a \in \mathbb{N})$,

$$
c_{p^{a}}(k)=\left\{\begin{array}{l}
p^{a}-p^{a-1}, \text { if } p^{a} \mid k, \\
-p^{a-1}, \text { if } p^{a-1} \mid k, p^{a} \nmid k, \\
0, \text { if } p^{a-1} \nmid k .
\end{array}\right.
$$

Also, $c_{n}(k)$ is multiplicative as a function of two variables, i.e., considered as the function $c: \mathbb{N}^{2} \rightarrow \mathbb{Z}$, $c(k, n)=c_{n}(k)$. The inequality $\left|c_{n}(k)\right| \leq \operatorname{gcd}(k, n)$ holds for any $k, n \in \mathbb{N}$.

These and other general accounts of Ramanujan sums can be found in the books by Apostol [6], McCarthy [12], Schwarz and Spilker [19], Sivaramakrishnan [20].

\section{The function $E$}

The following results were proved by Liskovets [10].

Proposition 1. ([10, Lemmas 2, 5, Prop. 4])

(i) The function $E$ is multiplicative (as a function of several variables).

(ii) Let $p^{a_{1}}, \ldots, p^{a_{r}}$ be any powers of a prime $p\left(a_{1}, \ldots, a_{r} \in \mathbb{N}\right)$. Assume that $a:=a_{1}=a_{2}=\ldots=$ $a_{s}>a_{s+1} \geq a_{s+2} \geq \ldots \geq a_{r} \geq 1(r \geq s \geq 1)$. Then

$$
E\left(p^{a_{1}}, \ldots, p^{a_{r}}\right)=p^{v}(p-1)^{r-s+1} h_{s}(p),
$$

where the integer $v$ is defined by $v=\sum_{j=1}^{r} a_{j}-r-a+1$ and

$$
h_{s}(x)=\frac{(x-1)^{s-1}+(-1)^{s}}{x}
$$

is a polynomial of degree $s-2$ (for $s>1$ ).

Note that Liskovets used the term semi-multiplicative function, but this is reserved for another concept, see for example [20, Ch. XI].

Corollary 2. ([10, Th. 8, Cor. 11])

(i) For any integers $m_{1}, \ldots, m_{r} \in \mathbb{N}$,

$$
E\left(m_{1}, \ldots, m_{r}\right)=\prod_{p \mid m} p^{v(p)}(p-1)^{r(p)-s(p)+1} h_{s(p)}(p),
$$


where $v(p)$ and $s(p)$, depending now on $p$ are the integers $v$ and $s$, respectively, defined in Proposition 1.

(ii) For $m_{1}=\ldots=m_{r}=m$,

$$
f_{r}(m):=E(m, \ldots, m)=m^{r-1} \prod_{p \mid m} \frac{(p-1) h_{r}(p)}{p^{r-1}} .
$$

We first give the following simple convolution representation for the function $E$.

Proposition 3. For any $m_{1}, \ldots, m_{r} \in \mathbb{N}$,

$$
E\left(m_{1}, \ldots, m_{r}\right)=\sum_{d_{1}\left|m_{1}, \ldots, d_{r}\right| m_{r}} \frac{d_{1} \cdots d_{r}}{\operatorname{lcm}\left[d_{1}, \ldots, d_{r}\right]} \mu\left(m_{1} / d_{1}\right) \cdots \mu\left(m_{r} / d_{r}\right) .
$$

Proof. Using formula (9),

$$
\begin{gathered}
E\left(m_{1}, \ldots, m_{r}\right)=\frac{1}{M} \sum_{k=1}^{M} \sum_{d_{1} \mid \operatorname{gcd}\left(k, m_{1}\right)} d_{1} \mu\left(m_{1} / d_{1}\right) \cdots \sum_{d_{r} \mid \operatorname{gcd}\left(k, m_{r}\right)} d_{r} \mu\left(m_{r} / d_{r}\right) \\
=\frac{1}{M} \sum_{d_{1}\left|m_{1}, \ldots, d_{r}\right| m_{r}} d_{1} \mu\left(m_{1} / d_{1}\right) \cdots d_{r} \mu\left(m_{r} / d_{r}\right) \sum_{1 \leq k \leq M, d_{1}\left|k, \ldots d_{r}\right| k} 1,
\end{gathered}
$$

where the inner sum is $\sum_{1 \leq k \leq M, \operatorname{lcm}\left[d_{1}, \ldots, d_{r}\right] \mid k} 1=M / \operatorname{lcm}\left[d_{1}, \ldots, d_{r}\right]$, ending the proof.

By Möbius inversion we obtain from (15),

Corollary 4. For any $m_{1}, \ldots, m_{r} \in \mathbb{N}$,

$$
\sum_{d_{1}\left|m_{1}, \ldots, d_{r}\right| m_{r}} E\left(d_{1}, \ldots, d_{r}\right)=\frac{m_{1} \cdots m_{r}}{\operatorname{lcm}\left[m_{1}, \ldots, m_{r}\right]} .
$$

Formula (15) has also a number of other applications:

Application 5. Formula (15) shows that $E$ is integral valued and that it does not depend on $M$, so in (1) one can take $M=m$, the lcm of $m_{1}, \ldots, m_{r}$, remarked also by Liskovets [10, Section 1].

Application 6. If $m_{1}, \ldots, m_{r}$ are pairwise relatively prime, then $E\left(m_{1}, \ldots, m_{r}\right)=\varepsilon^{(r)}\left(m_{1}, \ldots, m_{r}\right)$, defined by (8). Indeed, in this case $\operatorname{lcm}\left[d_{1}, \ldots, d_{r}\right]=d_{1} \cdots d_{r}$ for any $d_{i} \mid m_{i}(1 \leq i \leq r)$ and the claim follows from (15) using that $\sum_{d \mid n} \mu(d)=\varepsilon(n)$.

Application 7. Also, (15) furnishes a simple direct proof of the multiplicativity of $E$. Note that Liskovets [10] used other arguments to show the multiplicativity. Observe that, according to (15), E is the convolution of the functions $F$ and $\mu^{(r)}$, where $F$ is given by

$$
F\left(m_{1}, \ldots, m_{r}\right)=\frac{m_{1} \cdots m_{r}}{\operatorname{lcm}\left[m_{1}, \ldots, m_{r}\right]} .
$$

Since $F$ and $\mu^{(r)}$ are multiplicative, $E$ is multiplicative too.

Application 8. Formula (15) leads to a simple direct proof of the orthogonality property (4). Using the Gauss formula $\sum_{d \mid n} \phi(d)=n$,

$$
\begin{gathered}
E\left(m_{1}, m_{2}\right):=\frac{1}{M} \sum_{k=1}^{M} c_{m_{1}}(k) c_{m_{2}}(k)=\sum_{d_{1}\left|m_{1}, d_{2}\right| m_{2}} \frac{d_{1} d_{2} \mu\left(m_{1} / d_{1}\right) \mu\left(m_{2} / d_{2}\right)}{\operatorname{lcm}\left[d_{1}, d_{2}\right]} \\
=\sum_{d_{1}\left|m_{1}, d_{2}\right| m_{2}} \mu\left(m_{1} / d_{1}\right) \mu\left(m_{2} / d_{2}\right) \operatorname{gcd}\left(d_{1}, d_{2}\right)=\sum_{d_{1}\left|m_{1}, d_{2}\right| m_{2}} \mu\left(m_{1} / d_{1}\right) \mu\left(m_{2} / d_{2}\right) \sum_{\delta \mid \operatorname{gcd}\left(d_{1}, d_{2}\right)} \phi(\delta) \\
=\sum_{\delta a k=m_{1}, \delta b \ell=m_{2}} \mu(k) \mu(\ell) \phi(\delta)=\sum_{\delta u=m_{1}, \delta v=m_{2}} \phi(\delta) \sum_{a k=u} \mu(k) \sum_{b \ell=v} \mu(\ell),
\end{gathered}
$$

where one of the inner sums are zero, unless $u=v=1$ and obtain that $E\left(m_{1}, m_{2}\right)=\phi(m)$ for $m_{1}=$ $m_{2}=m$ and $E\left(m_{1}, m_{2}\right)=0$ otherwise. 

1.

Now we derive another identity for $E$ which furnishes an alternative proof of formula (ii) in Proposition

Proposition 9. For any $m_{1}, \ldots, m_{r} \in \mathbb{N}$,

$$
E\left(m_{1}, \ldots, m_{r}\right)=\frac{1}{m} \sum_{d \mid m} c_{m_{1}}(d) \cdots c_{m_{r}}(d) \phi(m / d) .
$$

Proof. It follows from $(9)$ that $c_{n}(k)=c_{n}(\operatorname{gcd}(k, n))(k, n \in \mathbb{N})$. Observe that for any $i \in\{1, \ldots, r\}$, $\operatorname{gcd}\left(\operatorname{gcd}(k, m), m_{i}\right)=\operatorname{gcd}\left(k, \operatorname{gcd}\left(m, m_{i}\right)\right)=\operatorname{gcd}\left(k, m_{i}\right)$, since $m_{i} \mid m$, hence $c_{m_{i}}(k)=c_{m_{i}}\left(\operatorname{gcd}\left(k, m_{i}\right)\right)=$ $c_{m_{i}}\left(\operatorname{gcd}\left(\operatorname{gcd}(k, m), m_{i}\right)\right)=c_{m_{i}}(\operatorname{gcd}(k, m))$. We obtain

$$
E\left(m_{1}, \ldots, m_{r}\right)=\frac{1}{m} \sum_{k=1}^{m} c_{m_{1}}(\operatorname{gcd}(k, m)) \cdots c_{m_{r}}(\operatorname{gcd}(k, m)),
$$

and by grouping the terms according to the values $\operatorname{gcd}(k, m)=d$, where $d \mid m, k=d j, 1 \leq j \leq m / d$, $\operatorname{gcd}(j, m / d)=1$, we obtain $(17)$.

Application 10. By (17), with the notation of Proposition 1,

$$
E\left(p^{a_{1}}, \ldots, p^{a_{r}}\right)=\frac{1}{p^{a}} \sum_{d \mid p^{a}} c_{p^{a_{1}}}(d) \cdots c_{p^{a_{r}}}(d) \phi\left(p^{a} / d\right) .
$$

Using (10) we see that only two terms of (18) are nonzero, namely those for $d=p^{a}$ and $d=p^{a-1}$. Hence,

$$
\begin{gathered}
E\left(p^{a_{1}}, \ldots, p^{a_{r}}\right)=\frac{1}{p^{a}}\left(c_{p^{a_{1}}}\left(p^{a}\right) \cdots c_{p^{a_{r}}}\left(p^{a}\right) \phi(1)+c_{p^{a_{1}}}\left(p^{a-1}\right) \cdots c_{p^{a_{r}}}\left(p^{a-1}\right) \phi(p)\right) \\
=\frac{1}{p^{a}}\left((p-1) p^{a_{1}-1} \cdots(p-1) p^{a_{r}-1}+\left(-p^{a-1}\right)^{s}(p-1) p^{a_{s+1}-1} \cdots(p-1) p^{a_{r}-1}(p-1)\right),
\end{gathered}
$$

and a short computation gives formula (ii) in Proposition 1.

Consider the function $f_{r}(m)$ defined in Corollary 2 (case $m_{1}=\ldots=m_{r}=m$ ). Here $f_{1}=\varepsilon, f_{2}=\phi$.

Proposition 11. Let $r \geq 3$. The average order of the function $f_{r}(m)$ is $C_{r} m^{r-1}$, where

$$
C_{r}:=\prod_{p}\left(1+\frac{(p-1) h_{r}(p)-p^{r-1}}{p^{r}}\right) .
$$

More exactly, for any $0<\varepsilon<1$,

$$
\sum_{m \leq x} f_{r}(m)=\frac{C_{r}}{r} x^{r}+\mathcal{O}\left(x^{r-1+\varepsilon}\right) .
$$

Proof. The function $f_{r}$ is multiplicative and by (14),

$$
\sum_{m=1}^{\infty} \frac{f_{r}(m)}{m^{s}}=\zeta(s-r+1) \prod_{p}\left(1+\frac{(p-1) h_{r}(p)-p^{r-1}}{p^{s}}\right)
$$

for $s \in \mathbb{C}$, Re $s>r$, where the infinite product is absolutely convergent for $\operatorname{Re} s>r-1$. Hence $f_{r}=g_{r} * \mathrm{id}_{r-1}$ in terms of the Dirichlet convolution, where $g_{r}$ is multiplicative and for any prime power $p^{a}(a \in \mathbb{N})$,

We obtain

$$
g_{r}\left(p^{a}\right)= \begin{cases}(p-1) h_{r}(p)-p^{r-1}, & a=1 \\ 0, & a \geq 2\end{cases}
$$

$$
\sum_{m \leq x} f_{r}(m)=\sum_{d \leq x} g_{r}(d) \sum_{e \leq x / d} e^{r-1}=\frac{x^{r}}{r} \sum_{d \leq x} \frac{g_{r}(d)}{d^{r}}+\mathcal{O}\left(x^{r-1} \sum_{d \leq x} \frac{\left|g_{r}(d)\right|}{d^{r-1}}\right)
$$

and (20) follows by usual estimates. 


\section{The function $A$}

Consider now the function $A$ given by (5).

The next formulae are similar to (15) and (17). The following one was already given in [22, Section $3]$.

Proposition 12. For any $m_{1}, \ldots, m_{r} \in \mathbb{N}$,

$$
A\left(m_{1}, \ldots, m_{r}\right)=\sum_{d_{1}\left|m_{1}, \ldots, d_{r}\right| m_{r}} \frac{\phi\left(d_{1}\right) \cdots \phi\left(d_{r}\right)}{\operatorname{cm}\left[d_{1}, \ldots, d_{r}\right]},
$$

Proof. Similar to the proof of Proposition 3, this is obtained by inserting $\operatorname{gcd}\left(k, m_{i}\right)=\sum_{d_{i} \mid \operatorname{gcd}\left(k, m_{i}\right)} \phi\left(d_{i}\right)$ $(1 \leq i \leq r)$.

Corollary 13. The function A is multiplicative (as a function of several variables).

Proof. According to (21), $A$ is the convolution of the functions $G$ and the constant 1 function, where $G$ is given by

$$
G\left(m_{1}, \ldots, m_{r}\right)=\frac{\phi\left(m_{1}\right) \cdots \phi\left(m_{r}\right)}{\operatorname{lcm}\left[m_{1}, \ldots, m_{r}\right]},
$$

both being multiplicative. Therefore $A$ is also multiplicative.

Proposition 14. For any $m_{1}, \ldots, m_{r} \in \mathbb{N}$,

$$
A\left(m_{1}, \ldots, m_{r}\right)=\frac{1}{m} \sum_{d \mid m} \operatorname{gcd}\left(d, m_{1}\right) \cdots \operatorname{gcd}\left(d, m_{r}\right) \phi(m / d) .
$$

Proof. Similar to the proof of Proposition 9. Using that $\operatorname{gcd}\left(k, m_{i}\right)=\operatorname{gcd}\left(\operatorname{gcd}(k, m), m_{i}\right)(1 \leq i \leq r)$ we have

$$
A\left(m_{1}, \ldots, m_{r}\right)=\frac{1}{m} \sum_{k=1}^{m} \operatorname{gcd}\left(\operatorname{gcd}(k, m), m_{1}\right) \cdots \operatorname{gcd}\left(\operatorname{gcd}(k, m), m_{r}\right),
$$

and by grouping the terms according to the values $\operatorname{gcd}(k, m)=d$ we obtain the formula.

Corollary 15. Let $p$ be a prime and let $a_{1}, \ldots, a_{r} \in \mathbb{N}$. Assume that $a_{0}:=0<a_{1} \leq a_{2} \leq \ldots \leq a_{r}$. Then

$$
A\left(p^{a_{1}}, \ldots, p^{a_{r}}\right)=p^{a_{0}+a_{1}+\ldots+a_{r-1}}+\left(1-\frac{1}{p}\right) \sum_{\ell=1}^{r} p^{a_{0}+a_{1}+\ldots+a_{\ell-1}} \sum_{j=a_{\ell-1}}^{a_{\ell}-1} p^{(r-\ell) j} .
$$

Proof. Let $a:=a_{r}$. Then $\operatorname{lcm}\left[p^{a_{1}}, \ldots, p^{a_{r}}\right]=p^{a}$. From (22) we obtain

$$
\begin{aligned}
& A\left(p^{a_{1}}, \ldots, p^{a_{r}}\right)=\frac{1}{p^{a}} \sum_{j=0}^{a} \operatorname{gcd}\left(p^{j}, p^{a_{1}}\right) \cdots \operatorname{gcd}\left(p^{j}, p^{a_{r}}\right) \phi\left(p^{a-j}\right) \\
& =p^{a_{0}+a_{1}+\ldots+a_{r-1}}+\left(1-\frac{1}{p}\right) \sum_{j=0}^{a-1} p^{\min \left(j, a_{1}\right)+\ldots+\min \left(j, a_{r}\right)-j},
\end{aligned}
$$

where the last sum is

$$
\begin{gathered}
\sum_{j=0}^{a_{1}-1} p^{r j-j}+\sum_{j=a_{1}}^{a_{2}-1} p^{a_{1}+(r-1) j-j}+\ldots+\sum_{j=a_{r-1}}^{a_{r}-1} p^{a_{1}+a_{2}+\ldots+a_{r-1}+1 j-j} \\
=\sum_{\ell=1}^{r} \sum_{j=a_{\ell-1}}^{a_{\ell}-1} p^{a_{0}+a_{1}+\ldots+a_{\ell-1}+(r-\ell) j},
\end{gathered}
$$

finishing the proof. 
Application 16. From (21) we have for any $m_{1}, \ldots, m_{r} \in \mathbb{N}$,

$$
A\left(m_{1}, \ldots, m_{r}\right) \geq \sum_{d_{1}\left|m_{1}, \ldots, d_{r}\right| m_{r}} \frac{\phi\left(d_{1}\right) \cdots \phi\left(d_{r}\right)}{d_{1} \cdots d_{r}}=\sum_{d_{1} \mid m_{1}} \frac{\phi\left(d_{1}\right)}{d_{1}} \cdots \sum_{d_{r} \mid m_{r}} \frac{\phi\left(d_{r}\right)}{d_{r}}=A\left(m_{1}\right) \cdots A\left(m_{r}\right),
$$

cf. (6), with equality if $m_{1}, \ldots, m_{r}$ are pairwise relatively prime.

Note that if $m_{1}=\ldots=m_{r}=m$, then from its definition,

$$
A_{r}(m):=A(m, \ldots, m)=\frac{1}{m} \sum_{k=1}^{m}(\operatorname{gcd}(k, m))^{r}=\frac{1}{m} \sum_{d \mid m} d^{r} \phi(m / d),
$$

which is a multiplicative function. An asymptotic formula for $\sum_{m \leq x} A_{r}(m)$ was given by Alladi [3]. See also [22, Section 2].

A simple inequality for the functions $E$ and $A$ is given by

Proposition 17. For any $m_{1}, \ldots, m_{r} \in \mathbb{N}$,

$$
E\left(m_{1}, \ldots, m_{r}\right) \leq A\left(m_{1}, \ldots, m_{r}\right) .
$$

Proof. Using the inequality $\left|c_{n}(k)\right| \leq \operatorname{gcd}(k, n)$, mentioned in the Introduction, we obtain

$$
E\left(m_{1}, \ldots, m_{r}\right) \leq \frac{1}{m} \sum_{k=1}^{m}\left|c_{m_{1}}(k)\right| \cdots\left|c_{m_{r}}(k)\right| \leq \frac{1}{m} \sum_{k=1}^{n} \operatorname{gcd}\left(k, m_{1}\right) \cdots \operatorname{gcd}\left(k, m_{r}\right)=A\left(m_{1}, \ldots, m_{r}\right) .
$$

\section{Generalizations}

Let $f \in \mathcal{F}_{2}$ be a function of two variables and consider the function

$$
F_{f}\left(m_{1}, \ldots, m_{r}\right):=\frac{1}{m} \sum_{k=1}^{m} f\left(k, m_{1}\right) \cdots f\left(k, m_{r}\right) .
$$

Proposition 18. ([10, Lemma 5]) If $n \mapsto f(k, n)$ is multiplicative for any $k \in \mathbb{N}$ and $k \mapsto f(k, n)$ is periodic $(\bmod n)$ for any $n \in \mathbb{N}$, then $F_{f}$ is multiplicative.

Now suppose that $f$ has the following representation:

$$
f(k, n)=\sum_{d \mid \operatorname{gcd}(k, n)} g(d) h(n / d) \quad(k, n \in \mathbb{N})
$$

where $g, h \in \mathcal{F}_{1}$ are arbitrary functions. Functions $f$ defined in this way, as generalizations of the Ramanujan sums, were investigated in $[4,5]$. See also Apostol [6, Section 8.3].

Proposition 19. Assume that $f$ is given by (28). Then

i) $F_{f}$ has the representations

$$
\begin{gathered}
F_{f}\left(m_{1}, \ldots, m_{r}\right)=\sum_{d_{1}\left|m_{1}, \ldots, d_{r}\right| m_{r}} \frac{g\left(d_{1}\right) \cdots g\left(d_{r}\right)}{\operatorname{lcm}\left[d_{1}, \ldots, d_{r}\right]} h\left(m_{1} / d_{1}\right) \cdots h\left(m_{r} / d_{r}\right), \\
F_{f}\left(m_{1}, \ldots, m_{r}\right)=\frac{1}{m} \sum_{d \mid m} f\left(\operatorname{gcd}\left(d, m_{1}\right)\right) \cdots f\left(\operatorname{gcd}\left(d, m_{r}\right)\right) \phi(m / d) .
\end{gathered}
$$

ii) If $g$ and $h$ are multiplicative functions, then $F_{f}$ is multiplicative (as a function of several variables). 
Proof. i) Follows by the same arguments as in the proofs of Propositions 3, 9, 12 and 14.

ii) Direct consequence of (29).

The functions $E$ and $A$ are recovered choosing $g=\mathrm{id}, h=\mu$ and $g=\phi, h=1$, respectively, where note that $\operatorname{gcd}(k, n)=\sum_{d \mid \operatorname{gcd}(k, n)} \phi(d)$.

Now let $f(k, n)=\bar{f}(\operatorname{gcd}(k, n))(k, n \in \mathbb{N})$, where $\bar{f} \in \mathcal{F}_{1}$ is an arbitrary function. Then $f$ is of type (28) with $g=\bar{f} * \mu, h=\mathbf{1}$, since $\bar{f}(\operatorname{gcd}(k, n))=\sum_{d \mid \operatorname{gcd}(k, n)}(\bar{f} * \mu)(d)$.

Special choices of $\bar{f}$ can be considered. For $\bar{f}=$ id we reobtain the function $A$. As another example, let $\bar{f}=\tau$, with $g=h=\mathbf{1}$. Then we obtain

Corollary 20. The function $F_{\tau} \in \mathcal{F}_{r}$ is multiplicative and

$$
\begin{gathered}
F_{\tau}\left(m_{1}, \ldots, m_{r}\right)=\sum_{d_{1}\left|m_{1}, \ldots, d_{r}\right| m_{r}} \frac{1}{\operatorname{lcm}\left[d_{1}, \ldots, d_{r}\right]}, \\
F_{f}\left(m_{1}, \ldots, m_{r}\right)=\frac{1}{m} \sum_{d \mid m} \tau\left(\operatorname{gcd}\left(d, m_{1}\right)\right) \cdots \tau\left(\operatorname{gcd}\left(d, m_{r}\right)\right) \phi(m / d) .
\end{gathered}
$$

Further common generalizations of the functions $E$ and $A$ can be given using $r$-even functions. See $[12,19,23]$ for their definitions and properties.

\section{Linear congruences with constraints}

A direct generalization of the interpretation of $E$ given in the Introduction is the following. Let $M \in \mathbb{N}$ and let $\mathcal{D}_{k}(M)(1 \leq k \leq r)$ be arbitrary nonempty subsets of the set of (positive) divisors of $M$. For an integer $n$ let $N_{n}\left(M, \mathcal{D}_{1}, \ldots, \mathcal{D}_{r}\right)$ stand for the number of solutions $\left(x_{1}, \ldots, x_{r}\right) \in \mathbb{Z}_{M}^{r}$ of the congruence

$$
x_{1}+\ldots+x_{r} \equiv n(\bmod M)
$$

satisfying $\operatorname{gcd}\left(x_{1}, M\right) \in \mathcal{D}_{1}, \ldots, \operatorname{gcd}\left(x_{r}, M\right) \in \mathcal{D}_{r}$.

If $\mathcal{D}_{k}=\left\{M / m_{k}\right\}(1 \leq k \leq r)$ and $n=0$, then we reobtain the function $E$.

Special cases of the function $N_{n}\left(M, \mathcal{D}_{1}, \ldots, \mathcal{D}_{r}\right)$ were investigated earlier by several authors.

The case $\mathcal{D}_{k}=\{1\}$, i.e., $\operatorname{gcd}\left(x_{k}, M\right)=1(1 \leq k \leq r)$ was considered for the first time by Rademacher [17] in 1925 and Brauer [7] in 1926. It was recovered by Nicol and Vandiver [16] in 1954, Cohen [8] in 1955, Rearick [18] in 1963, and others. The case $r=2$ was treated by Alder [1] in 1958.

The general case of arbitrary subsets $\mathcal{D}_{k}$ was investigated, among others, by McCarthy [11] in 1975 and by Spilker [21] in 1996. One has

$$
N_{n}\left(M, \mathcal{D}_{1}, \ldots, \mathcal{D}_{r}\right)=\frac{1}{M} \sum_{d \mid M} c_{M / d}(n) \prod_{i=1}^{r} \sum_{e \in \mathcal{D}_{i}(M)} c_{M / e}(d) .
$$

For $M=m, \mathcal{D}_{k}=\left\{m / m_{k}\right\}(1 \leq k \leq r)$ and $n=0$ (34) reduces to our Proposition 9.

The proof of formula (34) given in [21, Section 4], see also [12, Ch. 3], uses properties of $r$-even functions, Cauchy products and Ramanujan-Fourier expansions of functions.

See Chapter 3 of the book of McCarthy [12] for a survey of this topic.

It is well known that the number of solutions of polynomial congruences can be expressed using exponential sums, see for ex. [15, Th. 1.31]. Although the obtained expression can be easily transformed by means of Ramanujan sums in case of linear congruences with side conditions, this is not used in the literature cited in this Section.

In what follows we give a simple direct proof of (34) in the case $\mathcal{D}_{k}(M)=\left\{d_{k}\right\}(1 \leq k \leq r)$, applying the method of above. 
Proposition 21. Let $M \in \mathbb{N}, n \in \mathbb{Z}$ and let $d_{1}, \ldots, d_{r} \mid M$. Then

$$
\begin{gathered}
N_{n}\left(M,\left\{d_{1}\right\}, \ldots,\left\{d_{r}\right\}\right)=\frac{1}{M} \sum_{k=1}^{M} c_{M / d_{1}}(k) \cdots c_{M / d_{r}}(k) \exp (-2 \pi i k n / M) \\
=\frac{1}{M} \sum_{\delta \mid M} c_{M / d_{1}}(\delta) \cdots c_{M / d_{r}}(\delta) c_{M / \delta}(n) .
\end{gathered}
$$

Proof. Only the simple fact

$$
\sum_{k=1}^{M} \exp (2 \pi i k n / M)= \begin{cases}M, & M \mid n, \\ 0, & M \nmid n .\end{cases}
$$

and the definition of Ramanujan sums are required. By the definition of $N_{n}\left(M,\left\{d_{1}\right\}, \ldots,\left\{d_{r}\right\}\right)$,

$$
\begin{aligned}
N:= & N_{n}\left(M,\left\{d_{1}\right\}, \ldots,\left\{d_{r}\right\}\right)=\frac{1}{M} \sum_{\substack{1 \leq x_{1} \leq M \\
\operatorname{gcd}\left(x_{1}, M\right)=d_{1}}} \cdots \sum_{\substack{1 \leq x_{r} \leq M \\
\operatorname{gcd}\left(x_{1}, M\right)=d_{r}}} \sum_{k=1}^{n} \exp \left(2 \pi i k\left(x_{1}+\ldots+x_{r}-n\right) / M\right) \\
& =\frac{1}{M} \sum_{k=1}^{M} \exp (-2 \pi i k n / M) \sum_{\substack{1 \leq x_{1} \leq M \\
\operatorname{gcd}\left(x_{1}, M\right)=d_{1}}} \exp \left(2 \pi i k x_{1} / M\right) \cdots \sum_{\substack{1 \leq x_{r} \leq M \\
\operatorname{gcd}\left(x_{r}, M\right)=d_{r}}} \exp \left(2 \pi i k x_{r} / M\right),
\end{aligned}
$$

and denoting $x_{k}=d_{k} y_{k}, \operatorname{gcd}\left(y_{k}, M / d_{k}\right)=1(1 \leq k \leq M)$,

$$
\begin{gathered}
N=\frac{1}{M} \sum_{k=1}^{M} \exp (-2 \pi i k n / M) \sum_{\substack{1 \leq y_{1} \leq M / d_{1} \\
\operatorname{gcd}\left(y_{1}, M / d_{1}\right)=1}} \exp \left(2 \pi i k y_{1} /\left(M / d_{1}\right)\right) \cdots \sum_{\substack{1 \leq y_{r} \leq M / d_{r} \\
\operatorname{gcd}\left(y_{r}, M / d_{r}\right)=1}} \exp \left(2 \pi i k y_{r} /\left(M / d_{r}\right)\right) \\
=\frac{1}{M} \sum_{k=1}^{M} \exp (-2 \pi i k n / M) c_{M / d_{1}}(k) \cdots c_{M / d_{r}}(k)
\end{gathered}
$$

To obtain the second formula use that $c_{M / d_{i}}(k)=c_{M / d_{i}}(\operatorname{gcd}(k, M))$ and group the terms according to the values of $\operatorname{gcd}(k, M)=\delta$, cf. proof of Proposition 9 .

For $n=0$ and $d_{k}=M / m_{k}(1 \leq k \leq r)$ we reobtain the interpretation of the function $E$ and formula (17). We remark that the proof of Proposition 21 is a slight simplification of the proof of [13, Lemma 4.1].

\section{References}

[1] H. L. Alder, A generalization of the Euler $\phi$-function, Amer. Math. Monthly 65 (1958) 690-692.

[2] E. Alkan, A. Zaharescu and M. Zaki, Arithmetical functions in several variables, Int. J. Number Theory 1 (2005), 383-399.

[3] K. Alladi, On generalized Euler functions and related totients, in New Concepts in Arithmetic Functions, Matscience Report 83, Madras, 1975.

[4] D. R. Anderson, T. M. Apostol, The evaluation of Ramanujan's sums and generalizations, Duke Math. J., 20 (1953), 211-216.

[5] T. M. Apostol, Arithmetical properties of generalized Ramanujan sums, Pacific J. Math. 41 (1972), 281-293.

[6] T. M. Apostol, Introduction to Analytic Number Theory, Springer, 1976. 
[7] A. Brauer, Lösung der Aufgabe 30, Jber. Deutsch. Math.-Verein 35 (1926), 92-94.

[8] E. Cohen, A class of arithmetical functions, Proc. Nat. Acad. Sci. U.S.A. 41 (1955), 939-944.

[9] A. Deitmar, S. Koyama and N. Kurokawa, Absolute zeta functions, Proc. Japan Acad. Ser. A Math. Sci. 84 (2008), 138-142.

[10] V. A. Liskovets, A multivariate arithmetic function of combinatorial and topological significance, Integers 10 (2010), 155-177.

[11] P. J. McCarthy, The number of restricted solutions of some systems of linear congruences, Rend. Sem. Mat. Univ. Padova 54 (1975), 59-68.

[12] P. J. McCarthy, Introduction to Arithmetical Functions, Universitext, Springer, 1986.

[13] A. D. Mednykh and R. Nedela, Enumeration of unrooted maps with given genus, J. Combin. Theory, Ser. B 96 (2006), 706-729.

[14] N. Minami, On the random variable $\mathbb{N} \ni l \mapsto \operatorname{gcd}\left(l, n_{1}\right) \operatorname{gcd}\left(l, n_{2}\right) \cdots \operatorname{gcd}\left(l, n_{k}\right) \in \mathbb{N}$, Preprint, 2009, http://arxiv.org/abs/0907.0918v2

[15] W. Narkiewicz, Number Theory, World Scientific, Singapore, 1983.

[16] C. A. Nicol, H. S. Vandiver, A von Sterneck arithmetical function and restricted partitions with respect to a modulus, Proc. Nat. Acad. Sci. U.S.A. 40 (1954), 825-835.

[17] H. Rademacher, Aufgabe 30, Jber. Deutsch. Math.-Verein 34 (1925), 158.

[18] D. Rearick, A linear congruence with side conditions, Amer. Math. Monthly 70 (1963), 837-840.

[19] W. Schwarz and J. Spilker, Arithmetical Functions, London Mathematical Society Lecture Note Series, 184, Cambridge University Press, 1994.

[20] R. Sivaramakrishnan, Classical Theory of Arithmetic Functions, Monographs and Textbooks in Pure and Applied Mathematics, Vol. 126, Marcel Dekker, 1989.

[21] J. Spilker, Eine einheitliche Methode zur Behandlung einer linearen Kogruenz mit Nebenbedingungen, Elem. Math. 51 (1996), 107-116.

[22] L. Tóth, A survey of gcd-sum functions, J. Integer Sequences 13 (2010), Article 10.8.1, 23 pp.

[23] L. Tóth and P. Haukkanen, The discrete Fourier transform of $r$-even functions, 2010, submitted, http://arxiv.org/abs/1009.5281v1 